\title{
GLI INUIT DEL LABRADOR NELLE FONTI DOCUMENTARIE DEI SECOLI XVI - XVII - XVIII
}

\author{
Giulia Bogliolo Bruna \\ Jornalista - historiadora
}

RESUMO: Este artigo trata do tema do maravilhoso tendo como cenário o Pólo Norte, o mundo nórdico, "o império do frio", entre os séculos XVI e XVIII. Baseado em 13 documentos originais sobre os esquimós, busca retraçar a percepção do olhar europeu sobre $o$ aspecto físico, a vida social e a cultura material deste povo, até então desconhecido.

ABSTRACT: This article focuses on the subject of the merveilleux, having as backdrop the North Pole, the Nordic world, the "empire of the cold", between the $16^{\text {th }}$ and $17^{\text {th }}$ centuries. Based on 13 original documents concerning esquimos, this article retraces the European perception of the physical environment, social life and material culture of this people, until then unknown.

PALAVRAS-CHAVE: História Antropofágica, Esquimós, Maravilhoso, Cronistas, Imaginário.

KEY-WORDS: History Anthropophagic, Esquimos, Merveilleux, Cronicler, Imaginary.

Nec sit terris

ultima Tille

SENECA

\section{Il merveilleux nordique, monstra}

anthropomorpha, mirabilia ed umanità esotiche

Durante tutto il Medioevo, la rappresentazione cartografica é raffigurazione di un mondo sacro, pieno e stabile: l' Altro é ridotto a presenza esoti$\mathrm{ca}$, ineffabile. Nel Rinascimento, invece, lacunare ed ellittica, essa descrive un mondo in parte sconosciu- to, ed é strumento pragmatico che assegna all' $\mathrm{Al}-$ terità la funzione di offrirsi allo sguardo curioso dell' Osservatore. Dagli Atlanti Catalani al Planisfero di Ortelius, un merveilleux ancora di segno medievale definisce, metonimicamente, spazi 
geografici e mentali, mobili ed onirici, non ancora semantizzati.

Paese delle anime, della forza primordiale, della luce e dell' innocenza, il Settentrione é locus naturalis di beatitudine eterna e di un Paradiso immanente. Epifanie rivelatrici della prodigiosa varietas della Natura, monstra zoo-anthropomorpha e magnalia risorgono nelle Terrae Incognitae boreali, sede leggendaria di un meraviglioso polimorfo $\mathrm{e}$ pittoresco, che, non di rado, maschera, nelle carte, $i$ vuoti della Conoscenza.

Lo spoglio sistematico di un corpus documentario di 26 carte risalenti al Cinquecento (dalla Scoperta del Nuovo Mondo all' Atlas di Mercatore), Tab. 1, consente di rivelarc, mediante un' analisi incrociata, quantitativa e tematica, la ricchezza semantica e la stratificazione di sensi e sovrasensi che presiedono alla rappresentazione cartografica dell' Estremo Nord.

La Tab. 2 e la Fig. 1 consentono di quantificare la frequenza dei vari topoi e di valutarne l' incidenza nell' identificazione di una visione mitopoietica dell' universo boreale.

I merveilleux nordico si nutre della teratologia mutuata dalla tradizione giudaico-cristiana e di simboli: la cartografia popola quest' universo vergine ed esoterico di Grifoni, Acefali e, soprattutto, Pigmei, che lo definiscono, sostituendosi ai toponimi.

Se i Classici greci e latini avevano ubicato nelle immensità artiche gli Iperborei, comunità di uomini beati ed immortali, l' Europa Cristiana, nel tentativo di conciliare Sacre Scritture, disegni divini, scienza pagana ed esperienza, immagina un mondo coronato, al Nord, dal Paradiso Terrestre, con i suoi 4 fiumi, ed infestato dalle orde dell' Anticristo (MALAURIE, 1987, p. 117). Nella sua emblematica polivalenza di Altrove materiale e mistico, immanente $e$ trascendente al contempo, il Polo Nord é visto dalla geografia sacra del Rinascimento come il luogo-momento della Genesi e della Parusia. Miti secolari e sogni antichi sono i motori di viaggi alla scoperta "(du) lieu initial où le Créateur décida de faire naŝtre I" espèce humaine"'.

Nel suo trattato Des Merveilles du Monde, 1553, l' erudito Guillaume Postel identifica l' America notturna con il Regno degli Inferi, e, più tardi, nel Planisfero a proiezione polare del 1578, ubica al Polo il Paradiso Terrestre, circondato da quell' Oceano Glaciale, "où les autoctones communiquent de la Sibérie à l'Amérique par un pont de glace" (MALAURIE, 1987, p. 117). Mercatore raffigura al Polo una rupes nigra et altissima, dalla quale scaturiscono i quattro fiumi dell' Eden, che si gettano in un Oceano circumpolare. Pigmei alti quattro piedi e somiglianti a coloro che "in Groenlandia Screlingers vocant", vivono in queste regioni estreme.

Fedele alle tesi di Olaus Magnus, André Thevet; Cartografo del Re, attribuisce al Canada una natura vulcanica: il vulcanesimo é, allora, percepito come manifestazione tangibile della presenza di potenze demoniache. Gog e Magog, orde sataniche annunciate da Ezechelc, (38, 12-2), e dall' Apocalisse, (20, 7-8), Grifoni, Cinocefali, Balene a testa rossa, araldi del Maligno, migrano, per effetto del progredire delle conoscenze, in questo mondo singolare e fantastico, ove non esiste iato alcuno tra visibile ed invisibile, immanente $\mathrm{c}$ trascendente.

L' Isola dos Demonios e l' antica Tille di senechiana memoria costellano!' Occano Boreale, "padre di tutti i mostri", solcato da gigantesche montagne di ghiaccio, minacciose e spettrali. Lo scricchiolio della banchisa viene assimilato al lugubre lamento delle anime purganti, che, sinistro, sale da quell' Inferno bianco.

I. J. Magasich-Airola \& J.M. de Beer, America Magica. Quand l' Europe de la Renaissance croyail conquérir le Paradis, Parigi, 1994, p. 27. 
Metafora grafica di un' Alterità lontana e temuta, i monstra anthropomorpha materializzano, a quelle latitudini estreme, l' angoscia dei marinai e dei viaggiatori di fronte all' Ignoto ed alla Differenza:

peraltro é assai curioso [scrive Padre Charlevoix] che l' esitenza di uomini dotati di una sola gamba sia stata confermata... dal racconto di una Prigioniera appartenente alla tribù degli Eschimesi. Essa riferi' di aver visto, nel suo paese d' origine, Uomini di alta statura che evacuavano dalla bocca ed urinavano sotto le ascelle. Stando al suo racconto, presso gli Eschimesi vivrebbero individui difformi, che hanno una sola gamba, terminante in un piede smisurato. Ad ogni braccio avrebbero due mani. Queste creature avrebbero, inoltre, testa piatta, occhi e naso piccolissimi, in un corpo enorme (CHARLEVOIX, 1744, pp. 26-27).

Nella Cosmographie Universelle di Guillaume Le Testu, 1556, le creature favolose finiscono con l' indicare, "dans une Nouvelle France peuplée d" hommes à tête de chien ou de sanglier, des espaces à conquérir ..." (LESTRINGANT, 1991, p. 248). Durante il Cinquecento ed il Seicento, da spazio mitopoietico e sacro, il Nord diventa realtà economica da esplorare, conquistare, possedere: in ultima istanza, il mostro "c' est toujours l' Autre" (FAVIER, 1991, p. 239).

\section{Gli Inuit del Labrador: storia di una male-}

\section{encontre.}

Già nel corso del Quattrocento, i pescatori baschi, bretoni, normanni, irlandesi ed inglesi si avventurano nelle gelide acque dell' Oceano Boreale, spingendosi fino ai pescosi banchi delle Terre-Nuevas, toponimo generico con il quale si indicano l' Isola di Terranova e le coste del Labrador.

La cartografia registra nella Mer Ténébreuse la presenza d' isole ignote alla Tradizione greco-romana: l'Atlante di Bianco, 1436, raffigura Stockfixa, Isola del Merluzzo, (l' onomastico deriva dal vocabolo inglese stockfix, stoccafisso, appunto), che si puo' identificare, con una certa verosimiglianza, con Terranova, estremo limen dell' Atlantico: "Nul ne pense que c' est le début du Nouveau Monde" (FAVIER, 1991, p. 104). Alle elucubrazioni immaginifico-allegoriche di certa geografia misticheggiante, si oppone il sapere empirico dei morutiers, i primi Bianchi ad entrare in contatto con le Genti del Nord.

In quel tempo, la route des pêcheurs é la più attiva fra le rotte transatlantiche seguite dagli Europei: "bien avant celle qu' empruntait, au Sud, la prestigeuse Carrera des Indias, avec ses convois de galions vogant de Seville vers Carthagène et VeraCruz" (LESPAGNOL, 1991, p. 74).

In questo Empire du Froid, ricco di risorse faunistiche, gli Europei sviluppano un' intensa attività predatoria, combinando la pesca del merluzzo e la caccia ai mammiferi marini, foche e balene. Un documento del 1510 rivela che il commercio del merluzzo "faisait déjà l' objet d' une organisation méthodique avec un marché central à Rouen, où les marins écoulaient le gros de leur cargaison" (FRAIN, p. 90). Nel ventennio compreso tra il 1550 ed il 1575 , lo sfruttamento di questi giganteschi giacimenti aleutici mobilita, ogni anno, alcune centinaia di pescherecci francesi e non meno di 10000 marinai. $\mathrm{Da}$ Cadice a Bristol, la stragrande maggioranza dei porti europei che si affacciano sull' Atlantico partecipa a questa straordinaria avventura economica ed umana, nelle pescose acque della Mar de los Bacalaos. A partire dal XV secolo, la costa atlantica dell' odierno Canada si integra, attivamente, all' economia europea: durante l' estate, gli Eschimesi si recano nelle regioni antistanti lo Stretto di Belle Isle per procurarsi le mercanzie, mediante il baratto o il pillage.

Homo oeconomicus, ancora al paleolitico, l' Eschimese possiede un eccezionale istinto pragmatico ed utilitaristico: conservatore, egli é, pero', aperto, e pronto ad integrare, selettivamente, 
apporti esogeni nel suo corredo materiale. In questo modo, i manufatti europei, tessuti colorati, camicie, calderoni, tazze, coltelli, raschiatoi, entrano di prepotenza nel circuito commerciale degli Inuit del Labrador Settentrionale, antica Cultura di Thulé, favorendo un significativo transfert tecnologico.

Le suppellettili e gli utensili di uso quotidiano rinvenuti negli scavi in situ costituiscono insostituibili testimonianze dirette dei contatti tra Inuit del Labrador ed Europei in età storica. Ad csse si aggiungono, a complemento e conferma, gli elementi indiretti tratti dallo spoglio sistematico e quantitativo delle fonti europee. Esse annotano gli oggetti di uso comune, che talora si caricano di precisi significati simbolico-religiosi, in lunghe ed esaustive liste, che tradiscono la volontà dell' Osservatiore di procedere ad una ricognizione autoptica del' universo materiale inuk.

Nel presente lavoro, ci siamo valsi di un corpus di 13 relazioni, tutte originali e, per lo più, inedite, di cui 4 risalgono al ' 600 e 9 alla prima metà del 700 . La lista di questi documenti, corredata da indicazioni bibliografiche, é riportata in Tab. 3.

All' interno di precise coordinate spaziali, (l'area geografica presa in esame si limita alle regioni antistanti lo Stretto di Belle-Isle), abbiamo selezionato un échantillon, ristrelto, ma rappresentativo delle diverse tipologie di regard con cui l' Europeo percepi', interiorizzo', classifico' e descrisse l' Alterità inuk.

La schedatura del materiale ha privilegiato 4 nuclei tematici principali:

- l'aspetto fisico, il carattere, il fenotipo e gli elementi di vita sociale,

- le attività di sussistenza e gli scambi con gli Europei,

- gli oggetti culturalmente tipici, rivelazioni del genio attivo e creatore degli Inuit, che inventano, trasformano e perfezionano, inte- grando, selettivamente, le tecnologie esogene,

- i tratti distintivi della civiltà materiale, indagata anche nelle sue valenze sociopolitiche, rituali e religiose.

Le Tab. 4 e 5 e le Fig. 2 e 3 analizzano, quantificandola, la percezione che ebbero gli Europei dei tratti peculiari della società eschimese e dei rapporti interetnici che si stabilirono tra le due comunita. La Tab. 6 e la Fig. 4 recensiscono gli oggetti di uso più comune in epoca storica, compresi quelli di origine allogena. La Tab. 7 e la Fig. 5, infine, si concentrano sulla spiritualità, le tradizioni e la sensibilità artistica dei Sauvages du Nort.

All' occasione dei primi contatti, la comunicazione tra i gruppi é, necessariamente, affidata a mezzi non verbali, in primis, la gestualità. Osservatori acuti, gli Eschimesi imparano presto a decifrare il codice comportamentale dei Bianchi, giungendo a ritualizzare la fenomenologia dell' incontro: si avvicinano gridando espressioni di pace ed, avvalendosi di un impianto scenografico di sicuro effetto, agitano pelli di foca a mo' di bandiera.

Gli Europei, invece, sono sovente indotti in grossolani errori dalla lettura condizionata del comportamento degli Eschimesi: il fregarsi il ventre, manifestazione di gioia ed espressione di saluto secondo il costume inuk, viene interpretata come un' allusione ad un incipiente, ed auspicato, banchetto cannibalesco. Nel suo Joumal, Louis Jolliet, Signore di Anticosti c scopritore del Mississipi, demistifica questo topos, funzionale ad una percezione orientata ed a connotazione fortemente negativa: "Le donne attendevano fuori dalle capanne. Tutte erano visibilmente entusiaste dell' avvenimento, scrive, ma nesssuna esprimeva la sua gioia in modo sguaiato: parlavano soltovoce, sorridendo e fregandosi il ventre con le braccia e con le mani" (JOLLIET, 1993, p. 611). 
La frequentazione assidua, stagionale ed anonima dei pescatori europei e degli autoctoni favorisce, anche, la creazione di un pidgin francoinuk-montagnese, sorta di lingua franca, che facilita le operazioni di tratta. Nel 1743, il Sicur JeanLouis Fornel incontra, presso Capo Charles, un gruppo di Eschimesi che lo apostrofano cosi': "Tout Camara Troquo balena, non Characo", letteralmente: "Ehi, Amico, barattiamo della balena. Noi vogliamo la pace" (DORAIȘ, 1980, p. 168).

Nel corso del Cinquecento, ad una prima fase di contatti pacifici ed amichevoli, testimoniata, tra l' altro, da un importante meticciamento, fa seguito un periodo di diffidenza, se non proprio di ostilità aperta. Tuttc le fonti più antiche, (Biard, Champlain, Le Clerc, Lescarbot e Le Tac), riportano, in versioni che variano sulla fenomenologia dei fatti, ma concordano sul fondo, un incidente che sarebbe stato, a detta di tutti, all' origine della degenerazione dei rapporti tra le due comunità, provocando uno stato di endemica belligeranza:

Questa guerra [scrive Padre Biard] ha avuto inizio a causa del comportamento di alcuni Baschi, che si sarebbero resi colpevoli di un turpe ratto. Essi pagarono cara la loro incontinenza ... (Purtroppo, pero', non furono i soli, perché), gli Eschimesi sono, oggi, talmente inferociti che affronterebbero deliberatamente la morte pur di riuscire ad uccidere un nemico (BIARD, 1616, p. 68).

Stando alle testimonianze raccolte nel 1757 dal Vice Ammiraglio Thévenard, nel corso della sua inchiesta, l' inizio delle ostilità dovrebbe davvero esser ricondotto all' uccisione di una donna inuk ad opera di un marinaio maluino. Anche il gesuita Padre Silvy insiste sulla responsabilità dei Bianchi: "Sono convinto, scrive il missionario, che queste popolazioni nutrono una cosi' grande diffidenza nei nostri confronti a causa della crudeltà di cui abbiamo fatto prova in passato, tenendoli sempre alla larga dalle nostre imbarcazioni a colpi di fucile o, addirittura, a cannonate, e non esitando persino ad ucciderli" (SILVY, 1904, p. 58). Gli Eschimesi sono terrorizzati dalle armi da fuoco dei Francesi; quando le vedono, "tremano convulsamente e perdono sangue dal naso" (FORNEL, 1920-1921, p. 66).

Alla violenza gratuita dei Bianchi, essi rispondono con attacchi improvvisi e sanguinari, perturbando, di fatto, lo svolgimento delle operazioni di pesca nello Stretto di Belle Isle. Questi raids, fulminei, soprattutto notturni, permettono loro di procurarsi il ferro ed i manufatti europei, in particolare, coltelli, scialuppe c cordami.

Nella seconda metà del Seicento, il declino deli' industria baleniera dei Baschi favorisce l' espansione degli Uomini del Nord nelle regioni prospicienti il bacino del San Lorenzo: la presenza di insediamenti eschimesi nei territori compresi tra il Fiume SaintPaul c Blanc-Sablon é storicamente attestata nel periodo $1640-1690$.

I récits di religiosi e trafiquants concordano nel presentare gli Eschimesi come un popolo bellicoso, poco socievole, ma, soprattutto, pilleur, ladro: "barbares, féroces, farouches, défiants, inquiets".

L' Eschimese, in un processo di progressiva degenerazione, $\epsilon$, via via, assimilato alle furie infernali ed alle forze ctonie: "Ces démons", cosi' li apostrofa il Barone di Lahontan"2. Da Jean Alphonse, che li descrive ricorrendo a similitudini animalesche, "hanno code e grugno di porcelli", e, come le bestie,

2. La progressiva demonizzazione degli Inuit maschera il deterioramento della relazioni interetniche: l' Eschimese, che é uomo sensoriale, e quindi pronto a privilegiare il linguaggio del corpo, giunge persino a rifiutare il contatto fisico con $l^{\text {" }}$ Europeo: "Le marché conclu, ils reçoivent et donnent tout au bout d' un bâton". L.A. Lom d' Arce Baron de Lahontan, Voyages, L' Aja, 1703. Cfr. dal Tomo II, Mémoires de l' Amérique Septentrionale. Description Abrégée du Canada, $\mathrm{i}$ brani da noi tradotti e riportati in appendice all" articolo: "Les Sauvages de l' Enfer Blanc. L' immagine degli Inuit del Labrador nelle fonti documentarie francesi del Seicento", In: Miscellanea di Storia delle Esplorazioni, Genova, XVIII, pp. 109-110. 
"costruiscono i loro rifugi sottoterra", a Pietro Mattioli, che li taccia di idolatria e di barbarie, gli Hommes du Nort, sono per gli Europei archetipo di una sauvagerie estrema ed esorbitante: come Caino, sono esseri erranti e, come le fiere ed il Maligno, si annidano negli anfratti delle rocce.

Esotica anomia, I' inuk rimanda al caos primitivo delle Origini e si inscrive in un tempo primordiale, kerigmatico e profetico: in osmosi con la Natura é prodigium inquictante e misterioso. "Sans fois, ni lois", e, forse, dediti all' antropofagia, "ils se livrent dit-on au cannibalisme" (BIARD, p. 66), gli Eschimesi sono materiale antropologico ribelle e difficilmente omologabile: tra la civiltà ed il mondo indigeno lo iato é cosi' abissale che I' Animale interviene nelle descrizioni analogiche come intermediario indispensabile tra la Cultura, e la Natura. Per classificare questa nuova realtà, imprevista, ineffabile, tellurica, il giudizio normativo si avvale di un dispositivo triadico: l' Altro, l' Animale, l' Io, (I' Europeo).

\section{Un prodigium nell' Inferno bianco: la dolce inuk.}

Nella sua ambivalenza di archetipo e di realtà immanente, di curiosità senza nome e di metafora, la Sauvagesse du Nort, negata in quanto foriera di valori autonomi, designa, per metonimia, un Altrove primigenio e selvaggio, che puo', in potenza, essere apprivoisé, addomesticato.

E' soprattutto attraverso la mediazione della dolce e bella inuk, sensuale nelle sue forme rubensiane, ma pudica, disponibile, ma innocente,

3. Alphonse de Saincteonge, detto Jean de Fonteneau, (1544), "La Cosmographie avec I' Espère et le Régime du Soleil et du Nord". In P.G. Musset, a cura di, Recueil de Vyagers et Documents pour servir à l'Histoire de la Géograpie, Parigi, 1904, p. 179, "Les Voyages aventureux du capitaine Jean Alfonse Sainctongeois", Poitiers, 1559, p. 27. che l' Europeo é iniziato al vitale dualismo de! mistero sauvage.

Oggetto di duplice violenza, in quanto donna ed in quanto "indiana", la Sauvagesse borealc diventa l' instrumentum privilegiato di un progetto assimilazionista, che implica, al contempo, la reificazione, la mercificazione dell' Altro, ed il suo godimento in una logica esclusiva di dominio.

Privata della libertà fisica, nel par-deçà, l' inuk in cattività é "caricature chosifiéc et axiologisée".

La legenda dell' affichette stampata ad Augsburg da Mattheus Franker, primo documento etnostorico sugli Inuit del Labrador ${ }^{4}$, anticipa il tono delle relazioni che si sarebbero instaurate tra Europei ed autoctoni nel corso dei secoli.

La locandina, in lingua tedesca, racconta le circostanze del rapimento. Se il testo veicola, per ovvie ragioni pubblicitarie, un' immagine caricaturale ed a fosche tinte della donna, secondo la tradizionale triade: ferocia, licenziosità ed antropofagia, la raffigurazione iconica, la prima in assoluto di tipi cschimidi a carattere realistico, coniuga verità etnografica c manierismo. Lo iato tra la didascalia e la grafica si manifesta soprattutto nella descrizione del tatuaggio, attributo culturale significante nell' illustrazione, indizio di barbarie e di immoralità nello scritto: "I segni che porta sulle guance sono di colore blù. Si dice che sia il marito ad imprimerli sul volto della moglie all' atto del matrimonio, in segno di riconoscimento e di proprieta. In assenza di tale marchio, chiunque potrebbe liberamente accoppiarsi con lei, come fanno le bestie's.

La progressiva erotizzazione dell' immagine muliebre traspare dalle sequenze topiche dei capelli o nel richiamo sensuale della voce:

4. "True portrait of a savage woman with her little daughter in the district calted Nova Terra and brought to Antwerp and recently publicly seen there and still to be seen". Augsburg, 1567.

5. "True portrait ...", cit., (nostra traduzione). 
Le giovani donne acconciano $\mathrm{j}$ capelli in due piccoli chignons laterali, uno per parte, al di sopra delle orecchie. Il rimanente viene intrecciato a mo' di corona tutt' intorno al capo e termina, al centro, in un motivo che ricorda il bocciolo di una rosa. Questa acconciatura incornicia un viso sempre dolce e dall' incarnato roseo. La voce soave e delicata contribuisce ad accrescere il loro fascino, cui nessuno di noi rimase insensibile (JOLLIET, 1993, pp. 612-613).

La donna inuk é l' elemento catalizzatore di un processo di umanizzazione progressiva dell' Eschimese: autentica mediatrice culturale, essa, é, per l' Europeo, l' immagine archetipa e rassicurante della Terra-Madre, contrapposta alla fisicità prorompente ed inquietante della Sauvagesse tropicale, che, provocante, é allegoria della TerraOggetto. Il suo corpo nudo é epifania di una ben più temibile nudità morale. La donna inuk, invece, viene percepita, nella dialettica dell' Alterità e dell' Identificazione, attraverso un duplice gioco di negazioni, rispetto al Même, l' Europea, e rispetto a l' Autre, l' Indiana: essa sostituisce cosi', in positivo, l'Animale nella sua funzione di tramite tra la Cultura e la Natura.

Vestita con abiti ben confezionati, sovente impreziositi da graziosi inserti ornamentali, (la fabbrilità ed il senso estetico sono altrettanti, innegabili, indizi di cultura), e pudica, l' Eschimese é "maîtresse de son corps", ma osserva un codice comportamentale dettato dalla moralità naturáte: Sèla bella squaw é il simbolo vivente "de la source primitive de la sexualité" (JACQUIN, 1987, p. 189), la dolce inuk materializza, a latitudini estreme, l' ambiguo fantasma di un Eros trasgressivo, certo, ma paradossalmente innocente.

In questo Altrove pregno di segni non ancora decifrati, universo grandioso e terrificante di rocce e di ghiacci, la bella indigena é voce che umanizza lo spazio geografico: "Le loro voci sono molto più soavi di quelle delle altre Selvagge ...", scrive padre Silvy, peraltro non insensibile alla grazia delle Eschimesi: "Le donne eschimesi sono proporzionate, bianche, alte, grosse e grasse, con un viso grazioso, dolce, affabile e delicato. Tra queste genti vige la poligamia: le donne si occupano delle faccende domestiche" (SILVY, 1904, p.50). Conformemente ad una divisione tradizionale dei ruoli all' interno della famiglia, le Eschimesi si dedicano alle mansioni casalinghe ed alla concia delle pelli, ma sanno anche condurre "fort bien" l' umiak.

Nella dialettica dell' Alterità e dell' Identificazione, della diversità e dell' unicità del mondo, la bella inuk é metafora di un' umanità anteriore alla Rivelazione ed, Eva ante peccatum, si presenta, in una specularità inversa, come immagine relazionale alternativa dell' Europea:

Il loro seno [scrive Louis Jolliet] é sempre ben coperto $\mathrm{e}$, anche quando allattano, si guardano bene dal mostrarlo. Questo comportamento é certo più pudico di quello delle Francesi, le quali, soprattutto quando sono giovani spose, non esitano ad esibirlo in pubblico, per vantarsene (JOLLIET, 1993, p.612).

Alla frontiera tra Natura e Cultura, tra istinto e Ragione, tra fisicità e simbolo, l' Eschimese é singolarità sconcertante per la sua innata ortodossia, in un' Europa dilaniata dalle guerre di religione. Il manoscritto olandese Visbooc, redatto nel 1577-1578 da Adriaen Coenenzn, e consacrato alla fauna ittica ed ai mostri marini, riporta, con una certa enfasi, 1 ' - episodio di cui fu protagonista la prigioniera inuk, di cui si tratta nella locandina del 1566 :

La padrona ... [scrive Cocnenzn] si rivolgeva a questa Selvaggia, indicandole alcune statue, le une dorate, le altre policrome, ... e cosi' l' apostrofava: ' ... Prega. Ecco il vero Dio'. Mentre gliele additava, la. Selvaggia scosśe la testa, e levo' gli occhi al cielo in atteggiamento di preghiera. Alla luce dei fatti, non é affatto azzardato pensare che conoscesse il vero Dio, Signore dei Cieli (BRUNA, 1994, pp. 155-165). 
L' innata religiosità, e, più tardi, l' occasionale consumo di cibi europei agiscono da corollari nel processo di graduale riconoscimento dell' umanità eschimide. Da caricatura "chosifiée et axiologisée", a neofita, I'Eschimese viene ad incarnare, nell' immaginario collettivo europeo, l' archetipo della donna tellurica, femme à l' enfant, che puo' trasformare, con l' esempio, i Selvaggi del Nord, da esseri istintuali e ferini, a Hommes policés e religiosi, a conferma dell' unità adamitica del genere umano.

Altraverso il miracolo della Sauvagesse du Nort, che induce un riformato all' abiura del proprio credo, il gesuita Lallemant narra la storia di un' assimilazione, che comporta, per l' Eschimese, la rinuncia alla propria identità culturale ed implica un' adesione spontanea e naturale ai valori euro-cristiani dell' ordine, dell' autorità e della gerarchia.

Per i missionari, "à la conquête des âmes", il Grande Nord $\varepsilon$ Inferno bianco, infestato dalle furie demoniache e popolato da miscredenti. La conversione dell' Eschimese segna il passaggio da una Natura senza Dio ad una Natura epifania della Divinità, agendo da potente strumento provvidenziale per recuperare il fervore religioso e ripristinare I' unità ecclesiale perduta nelle diatribe confessionali. L' episodio della conversione riportato dal gesuita Charlevoix (CHARLEVOIX, 1744, p. 97), assurge a metafora csemplare di un' assimilazione riuscita ed, apparentemente, indolore.

Altro esempio più prosaico: l' adozione di costumi sessuali allogeni, in particolare il bacio à la française. Il bacio sulla bocca, che non rientra nelle pratiche erotiche degli Eschimesi, appare in epoca storica, in seguito alla frequentazione delle indigene con i marinai ed i pescatori europei. Anche il prestito delle donne, in origine pratica rituale di ospitalità, finisce con il trasformarsi in strumento privilegiato per favorire ed incrementare il commercio. Muta, cosi', to sguardo degli Inuit nei confronti delle loro compagne: lo scambio si riduce a mera forma di meretricio.
Mossa da un' immensa curiosità nei confronti dell' uomo bianco, convinta che le assicuri una prole sana e forte, la donna inuk stringe legami, talora non occasionali, con partners stranieri: "Un Europeo, che aveva fatto naufragio sulle coste, soggiorno' presso gli Inuit c sposo' un' indigena", si legge in un Anonimo ${ }^{6}$.

L' epilogo di queste unioni miste é sovente drammatico: "Un naufrago basco ... (fu) accolto dagli Inuit [scrive Le Clerc] che gli diedero in isposa un' indigena. Un giorno la uccise e scappo' a bordo di un battello francese" (LECLERC, 1910, p. 417).

A questo racconto fa eco la tragica vicenda di un' Eschimese, che, offesa nei suoi sentimenti più profondi, reagisce all' onta dell' abbandono sacrificando il figlio: “... Venuta a conoscenza della sua fuga, si reco' sulla spiaggia e, dopo avcrlo chiamato a lungo, inutilmente, rendendosi conto che non sarebbe tomato, prese il figlio che aveva avuto da lui, lo squarto' e getto' una parte dei brandelli di carne a terra, gli altri in mare"(SILVY, 1904, p. 59).

\section{IV - Epilogo}

Curiosità naturale e prodigium in un Altrove primordialc ed assoluto, fantasma antico dell' overdocility femminile e metafora rassicurante della Terra-Madre, la Sauvagesse du Nort, negata, violentata, assimilata e convertita, é vittima di una reificazione alienante nel rapporto dichiaratamente non paritetico con l' Europeo, che, per principio, esclude l' uguaglianza nella differenza.

Dalla donna maya data in pasto ai molossi, "parce qu' elle est femme non consentante et Indienne à la

6. Anonimo 1730 - 1732, "Mémoire en forme de lettre pour le bon homme Nicolas établit parmy les Esquimaux, in France", Archives des Colonies, MG-1, Série A 11-C, Vol. 22, Ottawa, Archives Publiques du Canada, p. 64. 


\section{Tabela 1 - Lista dei documenti cartografici esaminati}

1) Martin Behaim (1492), globo-fac-simile a colori del 1842; Parigi, B.N. Dpt. Cartes et Plans,

2) Giovanni Matteo Contarini, (1506), planisfero, Firenze; Londra, British Library,

3) Johannes Ruysch (1507), gravure su rame; Roma, Cool, Arthur - Holzeimer,

4) Martin Waldsemüller, (1507), "Universalis Cosmographia Secundum Ptholomei Traditionem Et Americi Vespuci Aliorumque Lustrationes”, gravure su legno, Strasbourg; Wortemberg, Schloss Wolfegg

5) Laurent Friess (1522), "Orbis typus universalis justa hydrographorum traditionem exactissime depicta"; Parigi,B.N. Dpt. Cartes et Plans,

6) Giovanni Vespucci ( 1524), “Totius Orbis Descriptio”, gravure su rame, Cambridge, Hougton Library, Università di Harvard,

7) Atlas Miller, (1516-1532); Parigi, B. N. Dpt. Cartes et Plans,

8) Oronce Fine, (1536), "Recens et integralis depictio", planisfero cordiforme, gravure su legno; Parigi, B. N. Dpt. Cartes et Plans,

9) Sebastiano Caboto (1544), planisfero, Anversa; Parigi, B. N. Dpt. Cartes et Plans,

10) Giacomo Gastaldi (1548), carta raccolta da G. B. Ramusio nel 1556 nel III volume delle Navigazioni e Viaggi, Venezia; Parigi, B. N. Dpt. Cartes et Plans,

11) Antonio Lafreri, (circa 1550), mappamondo bicordiforme, Roma; Parigi, B. N. Dpt. Cartes et Plans,

12) Pierre Apian et Gemma Frisius (1550), "Cosmographia", Anversa; Parigi, B. N. Dpt. Cartes et Plans,

13) Diogo Homen (1558), "Carta dell'Atlantico Settentrionale", manoscrtitto miniato; Londra, British Library",

14) Giovanni Francesco Camocio (1562), mappamondo, Venezia; Parigi, B. N. Dpt. Cartes et Plans,

15) Abraham Ortelius, (1564), mappamondo, gravure su rame; Londra, British Librany,

16) Guillaume Le Testu (1566), mappamondo, manoscrillo su pergamena; Parigi, B. N. Dpt. Cartes et Plans,

17) Bolognino Zaltieri (1566), "Carta dell'America del Nord”, Venezia; Parigi, B. N. Dpt. Cartes et Plans,

18) Olaus Magnus (1572), "Scandinavia", gravure; Parigi, B. N. Dpt. Cartes et Plans,

19) George Best (1758), "A True Discourse of the Late Voyage of Discoverie", carta universale di forma ovale, London; Parigi, B. N. Dpt. Cartes et Plans,

20) Denis de Rotis, (1579), "Carta marittima dell'Atlantico Settentrionale", manoscritto miniato su pergamena, Dieppe; Parigi, B. N. Dpt. Cartes et Plans,

21) John Dee (circa 1582), "Carta centrata sull'Atlantico"; Filadelfia, Biblioteca Libera di Filadelfia, Collection of Americana $n^{\circ} 42$,

22) Mickel Lock (1582), "Carta dell'emisfero Nord", pubblicata da Richard Hakluyt in Divers Voyages Touching the Discoverie of America, London; Parigi, B. N. Dpt. Cartes et Plans,

23) Jacopo Hondius (1589), "Vera totius expeditions naturae descriptio", Londra, Collezione Robert H. e Margareth C.,

24) Pedro de Lesmos (1590), manoscritto miniato su pergamena; Parigi, B. N. Dpt. Cartes et Plans,

25) Cornelis de Jode (1593), "America Pars Borealis", gravure su rame, Anversa; Chicago, Newberry Library,

26) Gerard Mercator (1595), "Carta del Polo Nord", gravure; Parigi, B. N. Dpt. Cartes et Plans. 
Tabela 2 - Analisi quantitativa dei documenti cartografici di Tab. 1: i topoi artici.

\begin{tabular}{|c|c|c|c|c|c|c|c|c|c|}
\hline & Frentum Anian & Gog e Magog & Tille & Monstra & Rupe Nigra C & Quattro Fiumi & Is. Demoni & Mare Getato & Iperborei \\
\hline 1 & & $*$ & $*$ & & & & & $*$ & $*$ \\
\hline 2 & & $*$ & & & & & & $*$ & $*$ \\
\hline 3 & & $*$ & & * & $*$ & $*$ & * & * & * \\
\hline 4 & & $*$ & * & & & & & * & \\
\hline 5 & & & & & & & & $*$ & \\
\hline 6 & & & & & & & & * & \\
\hline 7 & & & & * & & & $*$ & $*$ & $*$ \\
\hline 8 & & & & & $*$ & $*$ & * & $*$ & \\
\hline 9 & & & $*$ & 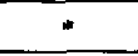 & & & & & $*$ \\
\hline 10 & & & & * & & & & & \\
\hline 11 & $*$ & & & & & & & & \\
\hline 12 & $*$ & & & 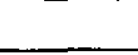 & & & & & \\
\hline 13 & $*$ & & & $*$ & & & & & \\
\hline 14 & 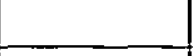 & & & $*$ & & & $*$ & & \\
\hline 15 & $*$ & & $*$ & * & & & & & \\
\hline 16 & & & & * & & & * & & \\
\hline 17 & $*$ & & & $*$ & & & & & \\
\hline 18 & & & $*$ & & & & & & \\
\hline 19 & $*$ & & & & & & & & \\
\hline 20 & $*$ & & & & & & & & \\
\hline 21 & * & & & & $*$ & $*$ & & & \\
\hline 22 & - & & & & & & * & & \\
\hline 23 & * & & & & & & & & \\
\hline 24 & & & & & * & $*$ & & & \\
\hline 25 & $*$ & $*$ & $*$ & & $*$ & * & $*$ & & * \\
\hline 26 & * & * & * & $*$ & * & $*$ & $*$ & & $*$ \\
\hline TOTALE & 11 & 6 & 7 & 10 & 6 & 6 & 8 & 9 & 7 \\
\hline
\end{tabular}


Tabela 3 - Lista dei documenti originali presi in esame per l'indagine quantitativa.

Anonimo 1720. Mémoires des Esquimatur, 1720, Ms. Col. 11 A $109 \mathrm{f} 60$, Archives Nationales de Paris: per la traduzione italiana, cfr. Giulia Bogliolo Bruna, Memorie sugli Eschimesi del Labrador in ll Polo, XLJ, 3/85, pp. 96-10I;

Anonimo 1722, Mémoire de ce que je şáai des Esquimaux en attendant une plus ample découverte, in France, Archives des Colonies MGI, Série C-11-A, vol. 122, Ottawa, Archives Publiques du Canada: 31-37;

Anonimo 1730 - 1732, Vie et Moeurs: De la manière de la religion esquimaux et de la situation de leur maison, in France, Archives des Colones MGI, Série C-11-A, vol. 122, Ottawa, Archives Publiques du Canada: 54-58;

Bacqueville de la Potherie, 1722, Histoire de l'Amérique Septentrionale ..., Parigi, 1722;

F. M. De Brouague, Divers mémoires de M. De Brouague au Conseil de la Marine, in Rapport de l'Archivist de la Province de Québcc pour 1922-1923, Québec, 1923;

Fornel J.-L., Découverte de la Baie des Esquimaux, in Rapport de l'Archiviste de la Province de Québec, Vol. I, (1920-1921): 60-75;

François R.-P, Letıre à M. Raudot Fils ("), datée du 20 octobre 1732, in France, Archives des Colonies MG-1, Série C-1 1-A, vol. 122, Ottawa, Archives Publiques du Canada: 43-47;

Jolliet L., Journal de Louis Jolliet allant à le descouverte de Labrador, pais des Esquimaux, 1694, Ms. 9275, Bibliotheqque Nationale, Nouvelles Acquisitions Françaises; per ta traduzione italiana di un'ampia scelta antologica, cfr. Giulia Bogliolo Bruna, ll Journal de Louis Jolliet allant à la desconverte de Labrador. pais des Esquimaux. Prima fonte etnostorica sugli Inuit del Labrador, in Columbeis V, Genova 1993, pp. 591-615;

Lahontan, L. A. Lom d'Arce, Barone di., Mêmoires de l'Amérique Septentrionale, ou la suite des Voyages de Mr. Le Baron de La Hontan, L'Aja, 1703; per la traduzione italiana della parte relativa agli Eschimesi del Labrador, (Tomo II, Description abregée du Canada), si rimanda a Giulia Bogliolo Bruna, Les Sawvages de l'Enfer Blanc. L'immagine degli Inuit del Labrador nelle fonti documentarie francesi del Seicento, in Miscellanea di storia delle esplorazioni XVIII, Genova, 1993, pp. 95-112;

Lallemant H., soprannominato dagli Uroni Archiendassé, Letrres envoiees de la Nouvelle France au R. P. Jacques Renault, Provincial de la Compagnie de Jesus en la Province de la France. par le R. P. Hierosme Lallemant. Supérteur des Missions de la dise Compagnie ence Nonveau Monde. Es année 1659; per la traduzione italiana, cfr. Giulia Bogliolo Bruna, Les Sauvages de L'Enfer Blanc ..., op. Cit., pp. 95-112;

Le Tac S., Histoire Cronologique de la Nouvelle France ou Canada depuis sa Découverte. (Mil Cinq Cents Quatre), jusques en l'an mil six cents trente deur. (incompiuta), pubblicata a Parigi nel 1888 da E. Réveillaud; per la traduzione italiana dei passi relativi agli Eschimesi del Labrador, cfr. Giulia Bogliolo Bruna, Les Sauvages de I'Enfer Blanc ..., op. cit., pp. 95-112;

Raudot A. D., lettera, in Rochemontejx Camille de, Relation par letsres de l'Amérique Septentrionale, (amnées 1709-1710). Paris, 1904.

Silvy A.. Journal d'un voiage depuis Bell' Isle jusques à Port Nelson, in Rélation par lettres de l'Amérique Septentrionale. P. Camille de Rochemonteix, (a cura di), Parigi, 1904. 
Tabela 4 - Attività di sussistenza e scambi con gli Europei. I documenti presi in esame sono quelli della Tabela 3.

\begin{tabular}{|c|c|c|c|c|c|}
\hline & Le Tac 1632 & Lallem. 1659 & Jolliet 1694 & B. Pot. 1697 & Lahont. 1709 \\
\hline Cacc/Pesc. & $*$ & 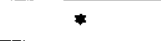 & $*$ & & $*$ \\
\hline Scambio & $*$ & & $*$ & 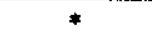 & $*$ \\
\hline \multirow[t]{2}{*}{ Rapina/Rub. } & & $\bullet$ & & & $\star$ \\
\hline & Raudot 1709 & Silvy 1709 & Brougg, 1717 & An. 1720 & An. 1722 \\
\hline Cace/Pesc. & 中 & $*$ & $*$ & $\star$ & $*$ \\
\hline \multicolumn{6}{|l|}{ Scambio } \\
\hline \multirow[t]{2}{*}{ Rapina/Rub. } & & & $*$ & & . \\
\hline & & An. 1730 & P. Fran. 1732 & Fornel 1743 & \\
\hline Cacc/Pesc. & & 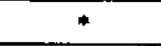 & & $*$ & \\
\hline \multicolumn{6}{|l|}{ Scambio } \\
\hline Rapina/Rub. & & & $*$ & $*$ & \\
\hline & & & & & \\
\hline
\end{tabular}

Tabela 5 - Aspetto, carattere ed elementi di vita sociale. I documenti presi in esame sono quelli di

\begin{tabular}{|c|c|c|c|c|c|}
\hline & Le Tac 1632 & Lallem. 1659 & Jolliet 1694 & B. Pot. 1697 & Lahont. 1709 \\
\hline Aspetto & & * & * & * & \\
\hline Carattere & * & & * & * & * \\
\hline Lingua & & & $*$ & & \\
\hline Famiglia & & & * & & \\
\hline \multirow[t]{2}{*}{ Vita sociale } & & & * & & * \\
\hline & Radout 1709 & Silvy 1709 & Brouag. 1717 & An. 1720 & An, 1722 \\
\hline Aspetto & $*$ & & * & & \\
\hline Carattere & & * & * & & \\
\hline \multicolumn{6}{|l|}{ Lingua } \\
\hline Famiglia & & * & * & * & $*$ \\
\hline \multirow[t]{3}{*}{ Vita sociale } & * & * & * & $*$ & * \\
\hline & & & & & \\
\hline & & An. 1730 & P. Fran. 1732 & Fornel 1743 & \\
\hline \multicolumn{6}{|l|}{ Aspetto } \\
\hline \multicolumn{6}{|l|}{ Carattere } \\
\hline Lingua & & & & * & \\
\hline Famiglia & & & * & & \\
\hline Vita sociale & & * & * & & \\
\hline
\end{tabular}


Tabela 6 - Elementi di cultura materiale. I documenti presi in esame sono quelli di Tabela 3.

\begin{tabular}{|c|c|c|c|c|c|}
\hline & Le Tac 1632 & Lallem. 1659 & Jolliet 1694 & B. Pot. 1697 & Lahont. 1709 \\
\hline Abitazione & & $*$ & $*$ & & \\
\hline Arred./Letti & & & $*$ & & \\
\hline Vasellame & & . & $*$ & & \\
\hline \multicolumn{6}{|l|}{ Lampada } \\
\hline Fuoco* & $*$ & 4 & $*$ & & \\
\hline Abbigliam. & $*$ & * & $*$ & $*$ & \\
\hline \multicolumn{6}{|l|}{ Occhiali } \\
\hline Tabacco & $*$ & & & & \\
\hline Alimentaz. & $*$ & * & $*$ & $*$ & \\
\hline Utensili & & $*$ & $*$ & & \\
\hline Armi & * & * & $*$ & & \\
\hline Imbarcaz. & $*$ & * & * & * & $*$ \\
\hline \multirow[t]{3}{*}{ Slitta/Cani } & & &.$^{*}$ & $*$ & \\
\hline & 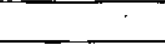 & & & & \\
\hline & Raudot 1709 & Sylvy 1709 & Brouag. 1717 & An. 1720 & An. 1722 \\
\hline Abitazione & * & $*$ & & $*$ & $*$ \\
\hline Arred /Letti & $*$ & & & & \\
\hline Vasellame & $*$ & $*$ & & $*$ & * \\
\hline Lampada & & & & & $*$ \\
\hline Fuoco* & $*$ & 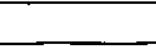 & & * & \\
\hline Abbigliam. & $*$ & $*$ & $*$ & $*$ & $*$ \\
\hline Occhiali & $*$ & & & & \\
\hline Tabacco & & & & & * \\
\hline Alimentaz. & $*$ & & $*$ & * & * \\
\hline Utensili & & & & * & $*$ \\
\hline Armi * & * & $*$ & * & $*$ & \\
\hline Imbarcaz. & $*$ & $*$ & $*$ & $*$ & $*$ \\
\hline \multirow[t]{3}{*}{ Slitta/Cani } & $*$ & * & * & & \\
\hline & & - & & & \\
\hline & & An. 1730 & P. Fran 1732 & Fornel 1743 & \\
\hline Abitazione & & $*$ & & & \\
\hline Arred./Letti & & * & & & \\
\hline Vascllame & & $*$ & & & \\
\hline Lampada & & $*$ & & & \\
\hline \multicolumn{6}{|l|}{ Fuoco } \\
\hline Abbiglian. & & * & $*$ & $*$ & \\
\hline Occhiali & & & & $*$ & \\
\hline \multicolumn{6}{|l|}{ Tabacco } \\
\hline Alimentaz. & & & & $*$ & \\
\hline Utensili & & $*$ & & & \\
\hline Armi & $*$ & $*$ & $*$ & & \\
\hline Imbarcaz. & & $*$ & $*$ & $*$ & \\
\hline Slitta/Cani & & . & & & \\
\hline
\end{tabular}


Tabela 7 - Spiritualità, tradizioni, sensibilità artistica. I documenti presi in esame sono quelli di Tabela 3.

\begin{tabular}{|c|c|c|c|c|c|}
\hline & Le Tac 1632 & Lallem. 1659 & Jolliet 1694 & B. Pot. 1697 & Lahont. 1709 \\
\hline \multicolumn{6}{|l|}{ Religione } \\
\hline \multicolumn{6}{|l|}{ Sciamani } \\
\hline \multicolumn{6}{|l|}{ Riti Funer. } \\
\hline \multirow[t]{3}{*}{ Arte } & & 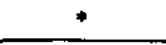 & & & \\
\hline & & & & & \\
\hline & Raudot 1709 & Silvy 1709 & Brouag 1717 & An. 1720 & An. 1722 \\
\hline Religione & & & $*$ & & $*$ \\
\hline Sciamani & & & $*$ & & $*$ \\
\hline Riti Funer. & $*$ & * & & & * \\
\hline \multicolumn{6}{|l|}{ Arte } \\
\hline & & & & & \\
\hline & & An. 1730 & P. Fran. 1732 & Fornel 1743 & \\
\hline \multicolumn{6}{|l|}{ Religione } \\
\hline & $*$ & $*$ & & & \\
\hline \multicolumn{6}{|l|}{ Sciamani } \\
\hline Riti Funer. & & & * & & \\
\hline Arte & & & & & \\
\hline
\end{tabular}

Figura 1 - Analisi quantitativa dei documenti cartografici di Tabela 1: i topoi artici.

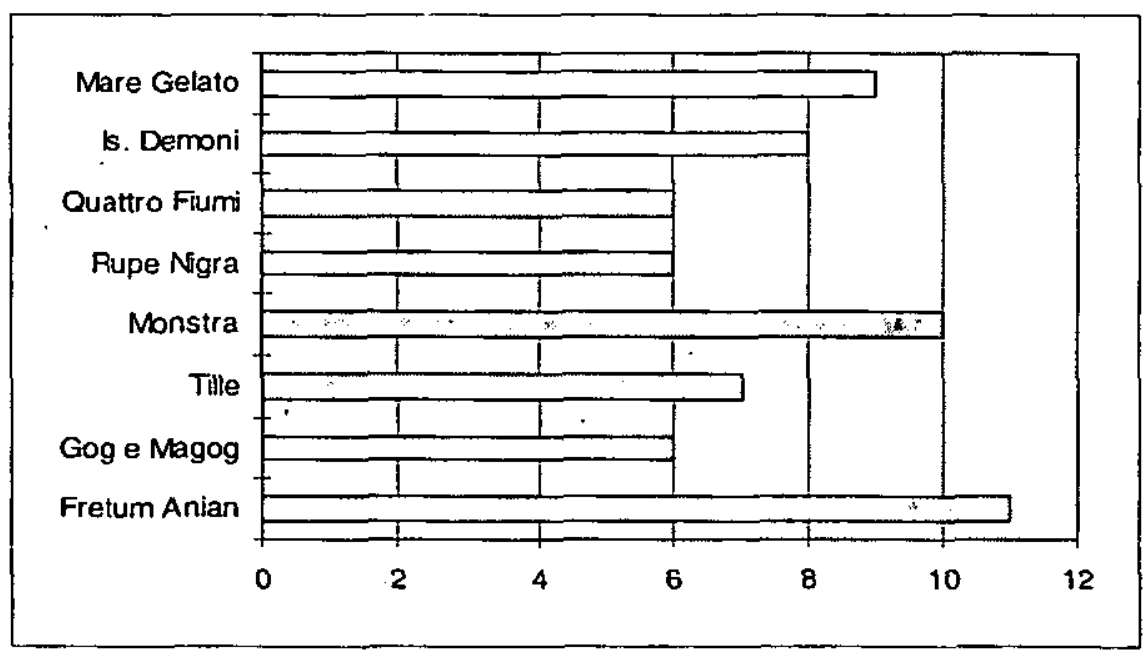




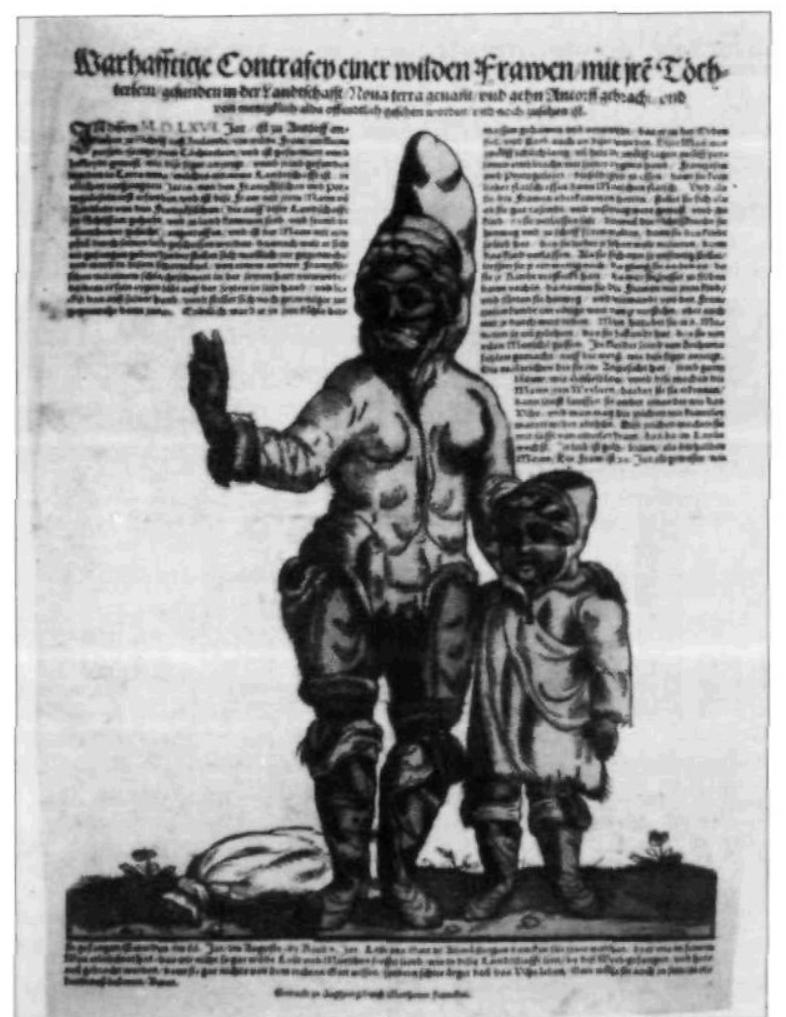

Figura 2
Figura 3

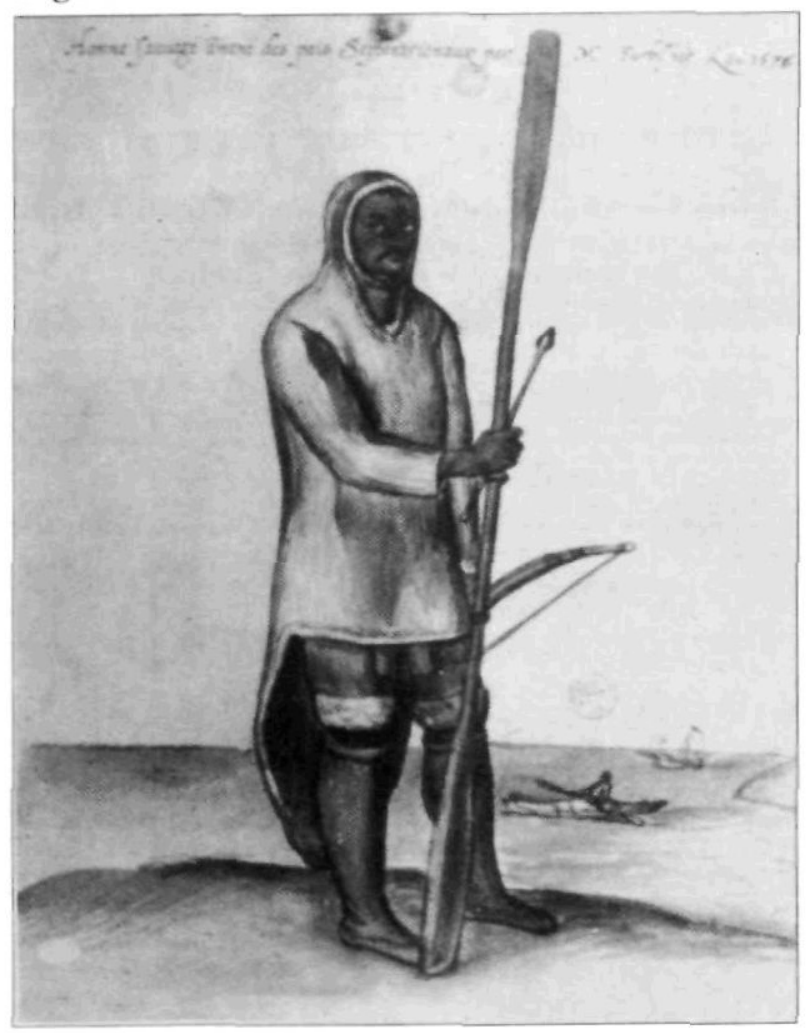


Figura 4 - Analisi quantitativa: elementi di cultura materiale. I documenti presi in esame sono quelli di Tabele 3.

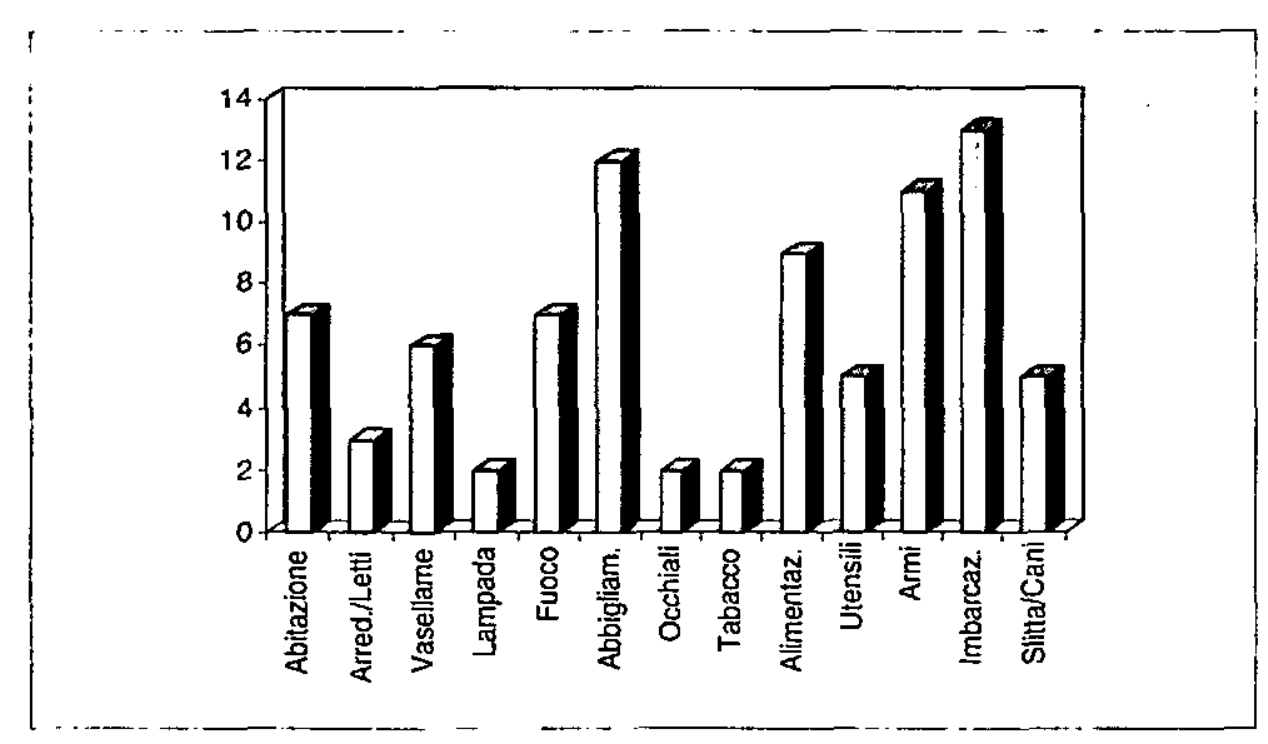

Figura 5 - Analisi quantitativa: spiritualità, tradizioni e sensibilità artistica. I documenti presi in esame sono qualli di Tabela 3.

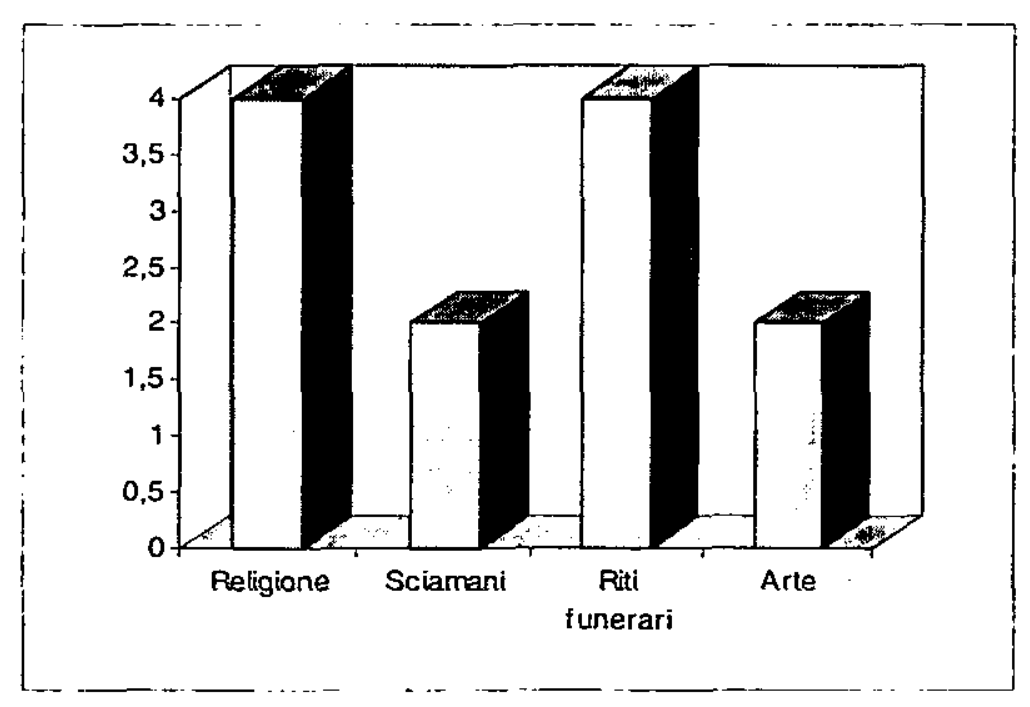


fois" (TODOROV, 1982, p. 307), alla sventurata Eschimese squartata viva dal chirurgo basco ${ }^{7}$, la storia della Conquista si compendia in forma paradigmatica, a tutte le latitudini, nel tragico destino, individuale e collettivo, dell' Indiana, figura

7. L' episodio é narrato da Sixte Le Tac in questi termini: "Sembra che le ostilità traggano origine dal crimine perpetrato da un chirurgo basco, il quale, dopo aver violentato un

\section{Bibliografie}

BIARD, P. In: Thwaites, (1896 - 1901). The Jesuit Relations and Allied Documents. Cleveland, 1616, vol. 3 .

BRUNA, Giulia Bogliolo. Visbooc: "Pesci, Mostri Marini e Savages of the Northe, una testimonianza etnostorica sugli Eschimesi in Europa, nella Il metà del Cinquecento". Miscellanea di Storia delle Esplorazioni, XIX, Genova. 1994, pp. 155-165.

CHARLEVOIX, F.X. Histoire et déscription générale de la Nouvelle France avec le Journal historique da voyage fait par ordre du Roi dans l' Amérique Septentrionale. Parigi, 1744, I.

DORAIS, L.-J. Dorais, "Les Inuit du Labrador Méridional: données linguistiques". In: Erudes Inuit Studies, Laval, 1980, vol. $4, n^{\prime \prime s} 1-2$.

FAVIER, J. Les grandes découvertes. D'Alexandre à Magellan. Parigi, 1991.

FORNEL, J.-L. "Découverte de la Baic des Esquimaux". In: Rapport de l'Archiviste de la Province du Québec, Vol. I. 1920-1921.

FRAIN, I. "L' épopée des Terte-Nuevas". In: $L$ ' Histoire, $n^{\circ} 36$. JACQUIN, P. Les Indiens Blancs. Parigi, 1987.

JOLLIET, L. "Journal de Loius Jolliet allant à la descouvente de emblematica di un' Alterità umana, "à la fois révélée et refusée" (TODOROV, 1982, p. 67).

Eschimese .... l' avrebbe squartata viva per estrarre dal suo ventre il feto che aveva concepito", LE TAC, S. Histoire chronologique de la Nouvelle France ou Canada depuis sa Découverte. (Mil Cinq Cents Quatre). jusques en l' an mil six cents trente deux, Parigi, 1888. Sugli Eschimesi, si vedano gli ampi stralci da noi riportati in appendice all" articolo, "Les sauvages de I' Enfer Blanc ...", cit., 108 - 109, (la citazione si trova a pagina 109).

Labrador, Paiis des Esquimaux, 1694". In: BRUNA, Giulia Boglioio. "Joumal de Louis Jolliet allant à la descouverte de Labrador, Païs Jes Esquimaux, 1694. La prima fonte etnostorica sugli Inuit del Labrador". In: Columbeis $V$, Genova, 1993.

LECLERC, C. In: GANONG, W.F. Le Clerc: New Relation of Gaspesia. Toronto, 1910.

LESPAGNOL, A. "Ah! Si les Français avaient conquis l' Amérique". In: $L$ 'Histoire, numero speciale pubblicato in occasione delle Celebrazioni Colombiane, $n^{\circ} 146$, giugno luglio 1991.

LESTRINGANT, F. "Le déclin d' un savoir. La crise de la cosmographie a la fin de la Renaissance". In: Annales, marzo-aprile, 199I.

MALAURIE, J. "Le Mythe du Pôle Nord: les Hyperboréens, Apollon, la Licorne de mer et l'Etoile Polaire". In: Pôle Nord 1983. Parigi, 1987.

SILVY, A. "Joumal d" un voyage depuis Bell' Isle jusqu' a Por Nelson". In: Relation par lettres de $L^{\prime}$ Amérique Septentrionale, P. Camille de Rochemonteix. Parigi, 1904.

TODOROV, T. La conquête de l'Amérique. La question de l'autre. Paris, 1982. 